\title{
Some new hints on cosmic-ray propagation from AMS-02 nuclei spectra
}

\author{
Jia-Shu Niu, ${ }^{1}$ and Hui-Fang Xue ${ }^{2}$ \\ ${ }^{1}$ Institute of Theoretical Physics, Shanxi University, Taiyuan, 030006, China \\ ${ }^{2}$ Department of Physics, Taiyuan Normal University, Taiyuan, 030619, China
}

\begin{abstract}
In this work, we considered 2 schemes (a high-rigidity break in primary source injections and a high-rigidity break in diffusion coefficient) to reproduce the newly released AMS-02 nuclei spectra $(\mathrm{He}, \mathrm{C}, \mathrm{N}, \mathrm{O}, \mathrm{Li}, \mathrm{Be}$, and $\mathrm{B})$ when the rigidity larger than $50 \mathrm{GV}$. The fitting results show that current data set favors a high-rigidity break at $\sim 325 \mathrm{GV}$ in diffusion coefficient rather than a break at $\sim 365 \mathrm{GV}$ in primary source injections. Meanwhile, the fitted values of the factors to rescale the cosmic-ray (CR) flux of secondary species/components after propagation show us that the secondary flux are underestimated in current propagation model. It implies that we might locate in a slow diffusion zone, in which the CRs propagate with a small value of diffusion coefficient compared with the averaged value in the galaxy. Another hint from the fitting results show that extra secondary CR nuclei injection may be needed in current data set. All these new hints should be paid more attention in future research.
\end{abstract}

\section{INTRODUCTION}

Cosmic-ray (CR) physics has entered a precisiondriven era (see, e.g., Niu and Li [1]). More and more fine structures have been revealed by a new generation of space-borne and ground-based experiments in operation. For nuclei spectra, the most obvious fine structure is the spectral hardening at $\sim 300 \mathrm{GV}$, which was observed by ATIC-2 [2], CREAM [3], PAMELA [4], and AMS-02 [5, 6].

Recently released AMS-02 nuclei spectra have confirmed that the spectral hardening exists not only in the primary $\mathrm{CR}$ nuclei species (He, $\mathrm{C}$, and $\mathrm{O}$ [7]), but also in the secondary $\mathrm{CR}$ nuclei species ( $\mathrm{Li}, \mathrm{Be}$, and $\mathrm{B}$ [8]) and hybrid nuclei species (N[9] [10]). This provides us an excellent opportunity to study the spectral hardening and the physics behind it quantitatively.

Some solutions are proposed to explain this observed phenomenon: (i) adding a new break in high-rigidity region $(\sim 300 \mathrm{GV})$ to the primary source injection spectra (see, e.g., Korsmeier and Cuoco [11, Boschini et al. [12, Niu et al. [13, Niu et al. [14, Zhu et al. [15, Niu et al. [16]); (ii) adding a new high-rigidity break in the diffusion coefficient (see, e.g., Niu et al. 16, Génolini et al. 17)); (iii) inhomogeneous diffusion (see, e.g., Blasi et al. [18, Tomassetti [19, 20, 21, Feng et al. 22, Guo and Yuan [23]); (iv) the superposition of local and distant sources (see, e.g., Vladimirov et al. 24], Bernard et al. 25], Thoudam and Hörandel 26, Tomassetti and Donato [27, Kachelrieß et al. [28], Kawanaka and Yanagita 29]).

The above explanations could be divided into two classes at first step: (i), (ii), and (iii), which ascribe the spectral hardening to non-local source effects; (iv), which ascribes it to the contribution of local sources. At second step, the first class of the first step could also be divided into two sub-classes: (i), which ascribes the spec-

\footnotetext{
* jsniu@sxu.edu.cn
}

tral hardening to the primary source injections; (ii) and (iii), which ascribe it to the propagation processes. If the spectral hardening comes from the CR sources, the ratio between secondary and primary species' spectra should appear featureless (or the primary and secondary spectra are equally hardened), since the secondary CR spectra inherit the features from the primary CR spectra. On the other hand, if the spectral hardening is due to the propagation processes, the ratio between secondary and primary species' spectra should be featured because the secondary species spectra not only inherit the hardening from the primary species (which is caused by the propagation of primary species), but are also hardened by their own propagation processes. This lead to a harder "tail" in CR secondary nuclei spectra than previous case (see, e.g., Fig. 2 in Niu et al. [16]). In this sense, (ii) and (iii) should have a similar prediction on the spectral hardening in primary and secondary nuclei spectra (see, e.g., Niu et al. [16, Feng et al. [22]).

As a result, in this work, we design 2 schemes to test the origin of the spectra hardening in CR nuclei spectra: (a) the spectral hardening comes from the sources, which can be described by a high-rigidity break in the primary source injections (Scheme I); (b) the spectral hardening comes from the propagation processes, which can be described by a high-rigidity break in the diffusion coefficient (Scheme II). Both of the schemes are implemented by the public code GALPROP v56 30 to reproduce the AMS-02 nuclei spectra in the global fitting. We hope that the AMS-02 data could give us a clear quantitative evidence to the origin of the spectral hardening in $\mathrm{CR}$ nuclei species.

\section{SETUPS}

As the framework has been established in our previous works [1, 13, 14, 16, we employ a Markov Chain Monte Carlo (MCMC) algorithm [31] which is embedded by GALPROP to do global fitting. The diffusionreacceleration model is used as the unique propagation 
model in this work. A uniform diffusion coefficient which depends on CR particles' rigidity is used in the whole propagation region. The propagation region is assumed to have a cylindrical symmetry and a free escape boundary condition. The radial $(r)$ and vertical $(z)$ grid steps are chosen as $\Delta r=1 \mathrm{kpc}$, and $\Delta z=0.2 \mathrm{kpc}$. The grid in kinetic energy per nucleon is logarithmic between $1 \mathrm{GeV}$ and $10^{4} \mathrm{GeV}$ with a step factor of 1.2 . The nuclear network used in our calculations is extended to silicon- 28 .

Some of the most important setups which are different from our previous work [16] are listed as follows (more detailed similar configurations could be found in Niu and Li [1], Niu et al. [16]):

(1) In this work, we do not use the proton and antiproton spectra in our global fitting. On the one hand, there is an obvious difference observed in the slopes of proton and other nuclei species when $Z>1$ [5] 7], which might indicate a different origin of the spectral hardening between proton and other primary CR species and needs to study independently. On the other hand, the spectrum of antiproton is dominately determined by proton spectrum, and might include some extra sources (like dark matter, see, e.g., Cui et al. [32, Cuoco et al. 33]). Excluding these data would help us to focus on the main aims of this work and avoid some unknown bias in the global fitting.

(2) The CR nuclei spectra are seriously influenced by solar modulation when the rigidity below 30 - 40 GV. Moreover, Aguilar et al. 34] has proved that the CR spectra of proton and helium are varying in solar cycle 24 when $R \lesssim 40 \mathrm{GV}$. At the same time, in AMS-02 nuclei spectra, data points from $1 \mathrm{GV}$ to $30 \mathrm{GV}$ always have small uncertainties, which seriously influence the global fitting results. Consequently, we use the data points above $50 \mathrm{GV}$ to do the global fitting, which could avoid the influences from low-rigidity data points and solar modulation model, and concentrate on the spectral hardening in high-rigidity region.

(3) Some of the free parameters which are not directly related to the high-rigidity spectra are removed or fixed as the best-fit values in our previous work [16. In detail, the low-rigidity slopes and breaks in primary source injections, all the solar modulation potentials $\left(\phi_{i} \mathrm{~s}\right)$, and all the parameters directly related to proton and antiproton spectra are removed. $D_{0}$ (the normalization of the diffusion coefficient), $z_{h}$ (the half-height of the propagation region), and $v_{A}$ (the Alfven velocity) are fixed.

(4) In this work, all the nuclei spectra data in the global fitting comes from AMS-02, which could avoid the complicities to combine the systematics from different experiments.

(5) In this work, the nitrogen spectrum (which is thought to be contributed both by primary and secondary components) is employed in the global fitting.

Altogether, the data set in our global fitting is

$$
\begin{aligned}
\boldsymbol{D}= & \left\{D_{\mathrm{He}}^{\mathrm{AMS}-02}, D_{\mathrm{C}}^{\mathrm{AMS}-02}, D_{\mathrm{N}}^{\mathrm{AMS}-02}, D_{\varnothing}^{\mathrm{AMS}-02},\right. \\
& \left.D_{\mathrm{Li}}^{\mathrm{AMS}-02}, D_{\mathrm{Be}}^{\mathrm{AMS}-02}, D_{\mathrm{B}}^{\mathrm{AMS}-02}\right\} .
\end{aligned}
$$

In Scheme I, the diffusion coefficient is parametrized as

$$
D_{x x}(R)=D_{0} \beta\left(\frac{R}{R_{0}}\right)^{\delta},
$$

where $\beta$ is the velocity of the particle in unit of light speed $c, R \equiv p c / Z e$ is the rigidity of a particle, and $R_{0}$ is the reference rigidity ( $4 \mathrm{GV})$.

For Scheme II, the diffusion coefficient is parametrized as

$$
D_{x x}(R)=D_{0} \cdot \beta\left(\frac{R_{\mathrm{br}}}{R_{0}}\right) \times\left\{\begin{array}{ll}
\left(\frac{R}{R_{\mathrm{br}}}\right)^{\delta_{1}} & R \leq R_{\mathrm{br}} \\
\left(\frac{R}{R_{\mathrm{br}}}\right)^{\delta_{2}} & R>R_{\mathrm{br}}
\end{array},\right.
$$

where $R_{\mathrm{br}}$ is the high-rigidity break, $\delta_{1}$ and $\delta_{2}$ are the diffusion slopes below and above the break.

The primary source injection spectra of all kinds of nuclei are assumed to be a broken power law form. In Scheme I, it is represented as:

$$
q_{\mathrm{i}}=N_{\mathrm{i}} \times \begin{cases}\left(\frac{R}{R_{\mathrm{A}}}\right)^{-\nu_{\mathrm{A} 1}} & R \leq R_{\mathrm{A}} \\ \left(\frac{R}{R_{\mathrm{A}}}\right)^{-\nu_{\mathrm{A} 2}} & R>R_{\mathrm{A}}\end{cases}
$$

where i denotes the species of nuclei, $N_{\mathrm{i}}$ is the normalization constant proportional to the relative abundance of the corresponding nuclei, and $\nu_{\mathrm{A} 1} / \nu_{\mathrm{A} 2}$ for the nucleus rigidity $R$ in the region divided by the break at the highrigidity $R_{\mathrm{A}}$. In this work, all the nuclei are assumed to have the same value of injection parameters.

For Scheme II, we have

$$
q_{\mathrm{i}}=N_{\mathrm{i}} \times R^{-\nu_{\mathrm{A}}}
$$

which are described by a power law with an index $\nu_{\mathrm{A}}$.

In GALPROP, the primary source isotopic abundances are determined by fitting to the data from ACE at about $200 \mathrm{MeV} /$ nucleon, based on a special propagation model [35, 36. But this appears some discrepancies when fit to some new data (see, e.g., Jóhannesson et al. [37]), which covers high-energy regions. Consequently, in both of the 2 schemes, $c_{\mathrm{He}}^{\mathrm{pri}}, c_{\mathrm{C}}^{\mathrm{pri}}, c_{\mathrm{N}}^{\mathrm{pri}}$, and $c_{\mathrm{O}}^{\mathrm{pri}}$ are employed to rescale the default abundances of helium- $4\left(7.199 \times 10^{4}\right)$, carbon$12\left(2.819 \times 10^{3}\right)$, nitrogen-14 $\left(1.828 \times 10^{2}\right)$, and oxygen- 16 $\left(3.822 \times 10^{3}\right)$. 38

At the same time, some works (see, e.g., Niu and $\mathrm{Li}$ [1, Niu et al. [16]) show that if one wants to fit the CR 
secondary spectra successfully, one should employ factors to rescale the flux of them after propagation. For antiproton, this factor is always in the region 0.8-1.9 [1, 39, 40, and always interpreted as the uncertainties from the antiproton production cross section [1] 44]. In our previous work [16, we found that all these factors are systematically larger than 1.0. This confirmed the necessity to employ them in the global fitting and lead us to find the physics behind them. Consequently, in this work, $c_{\mathrm{Li}}^{\mathrm{sec}}$, $c_{\mathrm{Be}}^{\mathrm{sec}}, c_{\mathrm{B}}^{\mathrm{sec}}$, and $c_{\mathrm{N}}^{\mathrm{sec}}$ are employed to rescale the flux of the secondary $\mathrm{CR}$ nuclei species ( $\mathrm{Li}, \mathrm{Be}$, and $\mathrm{B}$ ), and the secondary component of $\mathrm{N}$.

In summary, the parameter set for Scheme I is

$$
\begin{aligned}
\boldsymbol{\theta}_{1}=\{ & \delta, R_{\mathrm{A}}, \nu_{\mathrm{A} 1}, \nu_{\mathrm{A} 2}, \mid \\
& c_{\mathrm{He}}^{\mathrm{pri}}, c_{\mathrm{C}}^{\mathrm{pri}}, c_{\mathrm{N}}^{\mathrm{pri}}, c_{\mathrm{O}}^{\mathrm{pri}}, \mid \\
& \left.c_{\mathrm{Li}}^{\mathrm{sec}}, c_{\mathrm{Be}}^{\mathrm{sec}}, c_{\mathrm{B}}^{\mathrm{sec}}, c_{\mathrm{N}}^{\mathrm{sec}}\right\},
\end{aligned}
$$

for Scheme II is

$$
\begin{aligned}
\boldsymbol{\theta}_{2}= & \left\{R_{\mathrm{br}}, \delta_{1}, \delta_{2}, \nu_{\mathrm{A}}, \mid\right. \\
& c_{\mathrm{He}}^{\mathrm{pri}}, c_{\mathrm{C}}^{\mathrm{pri}}, c_{\mathrm{N}}^{\mathrm{pri}}, c_{\mathrm{O}}^{\mathrm{pri}}, \mid \\
& \left.c_{\mathrm{Li}}^{\mathrm{sec}}, c_{\mathrm{Be}}^{\mathrm{sec}}, c_{\mathrm{B}}^{\mathrm{sec}}, c_{\mathrm{N}}^{\mathrm{sec}}\right\} .
\end{aligned}
$$

\section{FITTING RESULTS}

As in our previous works 1, 13, 14, 16, the MCMC algorithm is employed to determine the posterior probability distribution of the parameters (see in Tables I and II) in Scheme I and II. The best-fit results of all the employed nuclei spectra for the two schemes are collected in Fig. 1. The best-fit results and the corresponding residuals (represented by $\sigma_{\text {eff }}$ ) of the primary nuclei species for the two schemes are given in the Fig. 2, that of the secondary and hybrid nuclei species are shown in Figs. 3 and 4 . For the best-fit results of the global fitting, we get $\chi^{2} /$ d.o.f $=108.97 / 201$ for Scheme I and $\chi^{2} /$ d.o.f $=96.16 / 201$ for Scheme II.

Note that in the lower panel of sub-figures in Figs. 2 . 3. and 4, the $\sigma_{\text {eff }}$ is defined as

$$
\sigma_{\mathrm{eff}}=\frac{f_{\mathrm{obs}}-f_{\mathrm{cal}}}{\sqrt{\sigma_{\mathrm{stat}}^{2}+\sigma_{\mathrm{sys}}^{2}}},
$$

where $f_{\text {obs }}$ and $f_{\text {cal }}$ are the points which come from the observation and model calculation; $\sigma_{\text {stat }}$ and $\sigma_{\text {sys }}$ are the statistical and systematic standard deviations of the observed points. This quantity could clearly show us the deviations between the best-fit result and observed values at each point based on its uncertainty. Considering the correlations between different parameters, we could not get a reasonable reduced $\chi^{2}$ for each part of the data set independently. As a result, we present the $\chi^{2}$ for each part of the data set in Figs. 2, 3 , and 4.

Generally speaking, all the nuclei spectra can be well fitted in 2 schemes (the largest deviation is smaller than
$3 \sigma$, see in Figs. 2, 3 , and 4,). Because the 2 schemes have a same data set and number of parameters, they have the same degree of freedom and can be compared directly by $\chi^{2}$. From the best-fit results, we get $\Delta \chi^{2}=\chi_{\mathrm{I}}^{2}-\chi_{\mathrm{II}}^{2}=$ 12.81, which is a decisive evidence 45] of indicating that the current data set favors the Scheme II. Considering the traditional simple assumptions in Scheme I and II (assuming a simple broken power-law for injection spectra, a uniform isotropic diffusion coefficient in the whole propagation region, etc.), we could not get a definite conclusion that the origin of the spectral hardening in nuclei spectra comes from the propagation processes, but at least it shows a tendency that current data set favors a high-rigidity break in the diffusion coefficient. More precise spectra date points in high rigidity $(\geq 2 \mathrm{TV})$, especially that of the secondary nuclei species, could give us more concrete conclusions.

The boxplot 46 for the $c_{i} \mathrm{~s}$ in this work are shown in the lower panels of Fig. 5. For comparison, the corresponding results of our previous work [16], in which the entire AMS-02 nuclei data (including the data points < $50 \mathrm{GV}$ ) is used in the global fitting, are shown in the upper panels in Fig. 5. We want to emphasize that in both of these works, all the nuclei spectra are considered in a self-consistent way and all of them are related to each other intrinsically. The fitting results clearly show that we could not reproduce the spectra of secondary species self-consistently without the employment of $c_{i}^{\mathrm{sec}}$ s. Consequently, all the fitting results of $c_{i}^{\text {pri }} \mathrm{s}$ and $c_{i}^{\mathrm{sec}} \mathrm{S}$ should be taken seriously. In Fig. 5 it is clearly shown that, same as that in previous work, no matter in Scheme I or II, the values of $c_{i}^{\text {pri }}$ s are systematically smaller than 1.0, while the values of $c_{i}^{\mathrm{sec}} \mathrm{s}$ are systematically larger than 1.0. Moreover, the values of $c_{i}^{\text {pri }} \mathrm{s}$ in this work are almost the same as that in previous work if we have considered the fitting uncertaintes, while the values of $c_{i}^{\mathrm{sec}} \mathrm{s}$ in this work are systematically larger than that in previous work.

As the nuclear charge number increases, both $c_{i}^{\text {pri }}$ s and $c_{i}^{\mathrm{sec}} \mathrm{s}$ have smaller values except $c_{\mathrm{N}}^{\mathrm{pri}}$ and $c_{\mathrm{Be}}^{\mathrm{sec}}$, respectively. Because the CR spectrum of nitrogen is composed by both primary and secondary components and has relative large fitting uncertainties, we will not focus on the value of $c_{\mathrm{N}}^{\mathrm{pri}}$ in this work. From the point view of $c_{i}^{\mathrm{sec}} \mathrm{s}$, beryllium is the most special CR secondary species.

\section{DISCUSSION AND CONCLUSION}

In this work, we considered 2 schemes to reproduce the newly released AMS-02 nuclei spectra (He, C, N, O, $\mathrm{Li}, \mathrm{Be}$, and $\mathrm{B}$ ) when $R>50 \mathrm{GV}$. The fitting results show that current data set favors a high-rigidity break at $\sim 325 \mathrm{GV}$ in diffusion coefficient rather than a break at $\sim 365 \mathrm{GV}$ in primary source injections, which is consistent with the results obtained in Génolini et al. 17. Moreover, the fitted values of $c_{i}^{\text {pri }}$ s (which are the factors to rescale the default isotopic abundances of helium-4, 
TABLE I. Constraints on the parameters in set $\boldsymbol{\theta}_{1}$. The prior interval, best-fit value, statistic mean, standard deviation and the allowed range at $95 \% \mathrm{CL}$ are listed for parameters. With $\chi^{2} /$ d.o.f $=108.97 / 201$ for best-fit result.

\begin{tabular}{lllll}
\hline \hline ID & Prior & \multicolumn{2}{l}{$\begin{array}{l}\text { Best-fit } \\
\text { value }\end{array}$} & $\begin{array}{l}\text { Posterior mean and } \\
\text { Standard deviation }\end{array}$ \\
& range & range \\
\hline$\delta$ & {$[0.1,1.0]$} & 0.36 & $0.36 \pm 0.01$ & {$[0.34,0.38]$} \\
$R_{\mathrm{A}}(\mathrm{GV})$ & {$[200,800]$} & 365 & $370 \pm 79$ & {$[248,511]$} \\
$\nu_{\mathrm{A} 1}$ & {$[1.0,4.0]$} & 2.34 & $2.34 \pm 0.01$ & {$[2.32,2.36]$} \\
$\nu_{\mathrm{A} 2}$ & {$[1.0,4.0]$} & 2.24 & $2.23 \pm 0.03$ & {$[2.18,2.28]$} \\
\hline$c_{\mathrm{He}}^{\mathrm{pr}}$ & {$[0.1,5.0]$} & 0.655 & $0.655 \pm 0.005$ & {$[0.646,0.664]$} \\
$c_{\mathrm{C}}^{\mathrm{pr}}$ & {$[0.1,5.0]$} & 0.554 & $0.554 \pm 0.005$ & {$[0.545,0.562]$} \\
$c_{\mathrm{N}}^{\mathrm{pri}}$ & {$[0.1,5.0]$} & 0.808 & $0.809 \pm 0.067$ & {$[0.698,0.923]$} \\
$c_{\mathrm{O}}^{\mathrm{pri}}$ & {$[0.1,5.0]$} & 0.486 & $0.486 \pm 0.004$ & {$[0.480,0.493]$} \\
\hline$c_{\mathrm{Li}}^{\mathrm{sec}}$ & {$[0.1,5.0]$} & 1.94 & $1.94 \pm 0.09$ & {$[1.79,2.09]$} \\
$c_{\mathrm{Be}}^{\text {sec }}$ & {$[0.1,5.0]$} & 2.28 & $2.28 \pm 0.10$ & {$[2.12,2.45]$} \\
$c_{\mathrm{B}}^{\mathrm{sec}}$ & {$[0.1,5.0]$} & 1.45 & $1.45 \pm 0.06$ & {$[1.35,1.56]$} \\
$c_{\mathrm{N}}^{\text {sec }}$ & {$[0.1,5.0]$} & 1.11 & $1.11 \pm 0.10$ & {$[0.96,1.28]$} \\
\hline \hline
\end{tabular}

TABLE II. Constraints on the parameters in set $\boldsymbol{\theta}_{2}$. The prior interval, best-fit value, statistic mean, standard deviation and the allowed range at $95 \%$ CL are listed for parameters. With $\chi^{2} /$ d.o.f $=96.16 / 201$ for best-fit result.

\begin{tabular}{lllll}
\hline \hline ID & $\begin{array}{l}\text { Prior } \\
\text { range }\end{array}$ & $\begin{array}{l}\text { Best-fit } \\
\text { value }\end{array}$ & $\begin{array}{l}\text { Posterior mean and } \\
\text { Standard deviation }\end{array}$ & range \\
\hline$R_{\text {br }}(\mathrm{GV})$ & {$[200,800]$} & 325 & $331 \pm 70$ & {$[233,468]$} \\
$\delta_{1}$ & {$[0.1,1.0]$} & 0.36 & $0.37 \pm 0.01$ & {$[0.34,0.39]$} \\
$\delta_{2}$ & {$[0.1,1.0]$} & 0.26 & $0.26 \pm 0.03$ & {$[0.21,0.30]$} \\
$\nu_{\mathrm{A}}$ & {$[1.0,4.0]$} & 2.34 & $2.34 \pm 0.01$ & {$[2.32,2.36]$} \\
\hline$c_{\mathrm{He}}^{\mathrm{pri}}$ & {$[0.1,5.0]$} & 0.653 & $0.653 \pm 0.005$ & {$[0.645,0.661]$} \\
$c_{\mathrm{C}}^{\text {pri }}$ & {$[0.1,5.0]$} & 0.551 & $0.551 \pm 0.005$ & {$[0.543,0.560]$} \\
$c_{\mathrm{N}}^{\mathrm{pri}}$ & {$[0.1,5.0]$} & 0.815 & $0.805 \pm 0.067$ & {$[0.707,0.926]$} \\
$c_{\mathrm{O}}^{\mathrm{pri}}$ & {$[0.1,5.0]$} & 0.479 & $0.479 \pm 0.004$ & {$[0.473,0.485]$} \\
\hline$c_{\mathrm{Li}}^{\mathrm{sec}}$ & {$[0.1,5.0]$} & 2.23 & $2.25 \pm 0.11$ & {$[2.05,2.40]$} \\
$c_{\mathrm{Be}}^{\text {sec }}$ & {$[0.1,5.0]$} & 2.57 & $2.59 \pm 0.12$ & {$[2.37,2.76]$} \\
$c_{\mathrm{B}}^{\mathrm{sec}}$ & {$[0.1,5.0]$} & 1.65 & $1.67 \pm 0.08$ & {$[1.53,1.78]$} \\
$c_{\mathrm{N}}^{\text {sec }}$ & {$[0.1,5.0]$} & 1.21 & $1.27 \pm 0.15$ & {$[1.04,1.37]$} \\
\hline \hline
\end{tabular}

carbon-12, nitrogen-14, and oxygen-16 in GALPROP) are systematically smaller than 1.0 and consistent with the results in our previous work [16] within fitting uncertainties. While the fitted values of $c_{i}^{\mathrm{sec}} \mathrm{s}$ (which are the factors to rescale the CR flux of secondary species/components after propagation) are systematically larger than 1.0 and larger than the values obtained in our previous work [16, which includes the entire spectra data points in the global fitting.

In some of the previous works (see, e.g., Niu and Li [1, Niu et al. [13, Niu et al. [14, Lin et al. 39, Lin et al. [40, Yuan et al. 47]), the $\overline{\mathrm{p}}$ rescale factor $c_{\overline{\mathrm{p}}}^{\mathrm{sec}}$ always have a value of $\sim 1.3$, which has been explained to approximate the ratio of antineutron-to-antiproton production cross section [44. Similarly, all the other $c_{i}^{\mathrm{sec}} \mathrm{S}$ could be interpreted as the same origins. However, generally speaking, the production cross sections of these secondary species are energy dependent. In Figs. 1, 2,3 , and 4 , one can find that the fitting results are quit well in most of the cases. If this is the right explanation, it is quit unnatural that all these secondary species have energy independent corrections on their production cross sections. On the other hand, it is also quit unnatural that all the production cross sections of these secondary species have been underestimated simultaneously. As a result, this explanation could be excluded to some extend. At least, it could not be the dominate factor.

According to observing the extended emission around Geminga and PSR B0656+14 pulsar wind nebulae (PWNe), Abeysekara et al. 48, have found that the estimated CR diffusion coefficient $\left(D_{0}\right)$ are more than two orders of magnitude smaller than the typical value derived from the secondary/primary nuclei species in galactic CRs. It infers that there exists slower diffusion zone (SDZ) around PWN, which could be extended to CR sources [49. Some previous works [50, 51, show that positron excess in CRs could be explained by this twozone model. If we locate in such a SDZ [52, a smaller 

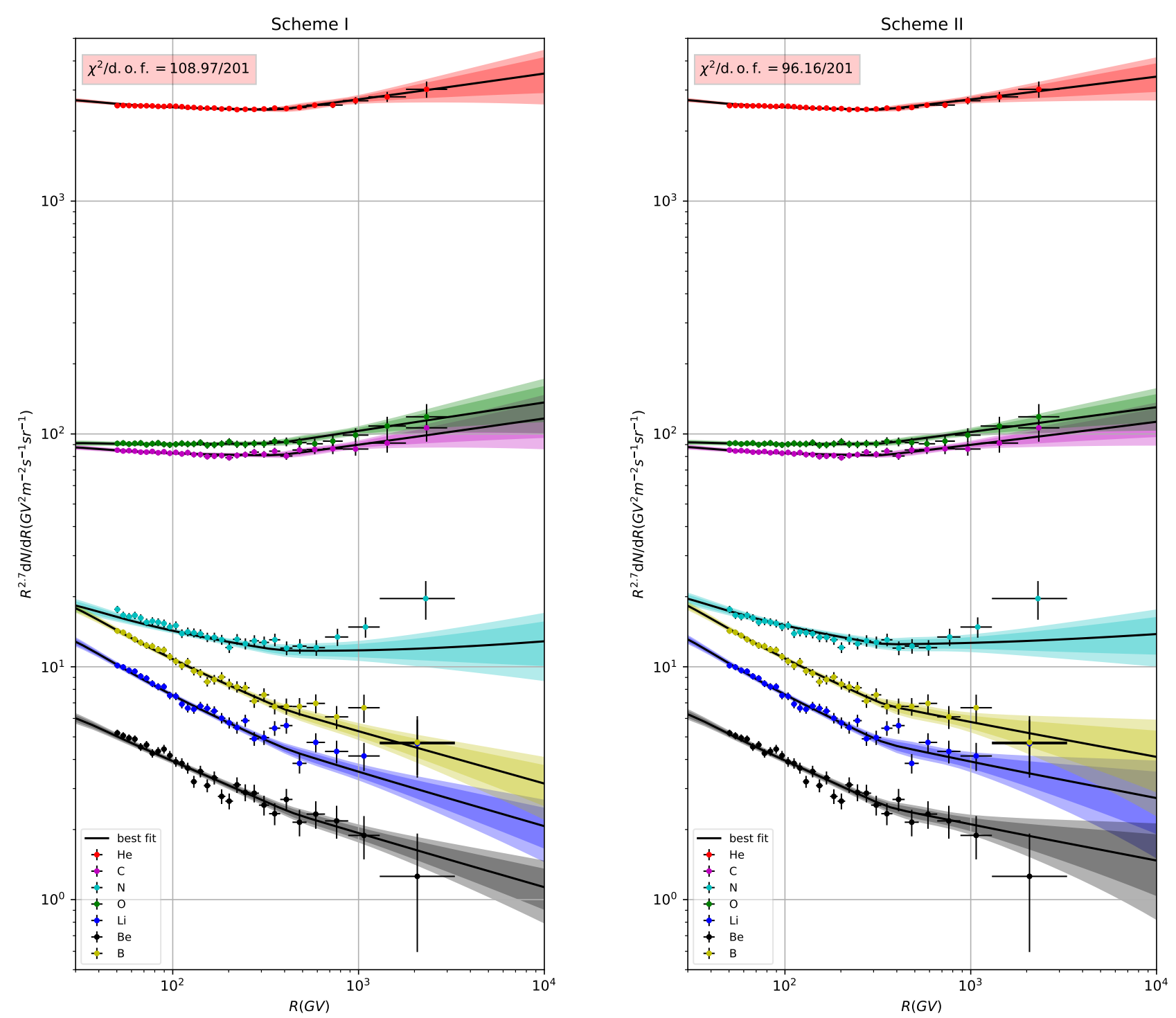

FIG. 1. The global fitting results of all the CR nuclei species employed in this work for Scheme I and II. The $2 \sigma$ (deeply colored) and $3 \sigma$ (lightly colored) bounds are also shown in the sub-figures. The relevant $\chi^{2} /$ d.o.f of the two schemes are also given in the sub-figures.

$D_{0}$ can lead to produce more secondary nuclei species' flux than the uniform diffusion in the whole galaxy. This could be the solution to the underestimation of secondary flux in current model.

Meanwhile, the $c_{i}^{\mathrm{sec}} \mathrm{s}$ in current work (averaged from $50 \mathrm{GV}$ to $2 \mathrm{TV}$ ) are systematically larger than that in our previous work (averaged from $2 \mathrm{GV}$ to $2 \mathrm{TV}$ ), which implies that it needs more secondary CR particles in high energy region to meet the observed data. 53 If it is the case, one needs extra injection of secondary nuclei species in high energy region. This scenario is recently studied by some interesting works (see, e.g., Yang and Aharonian [54] and Boschini et al. [55]).

In summary, we could ascribe the underestimation of the CR secondary flux to the SDZ which we locate in. At the same time, another hint implies it might need 

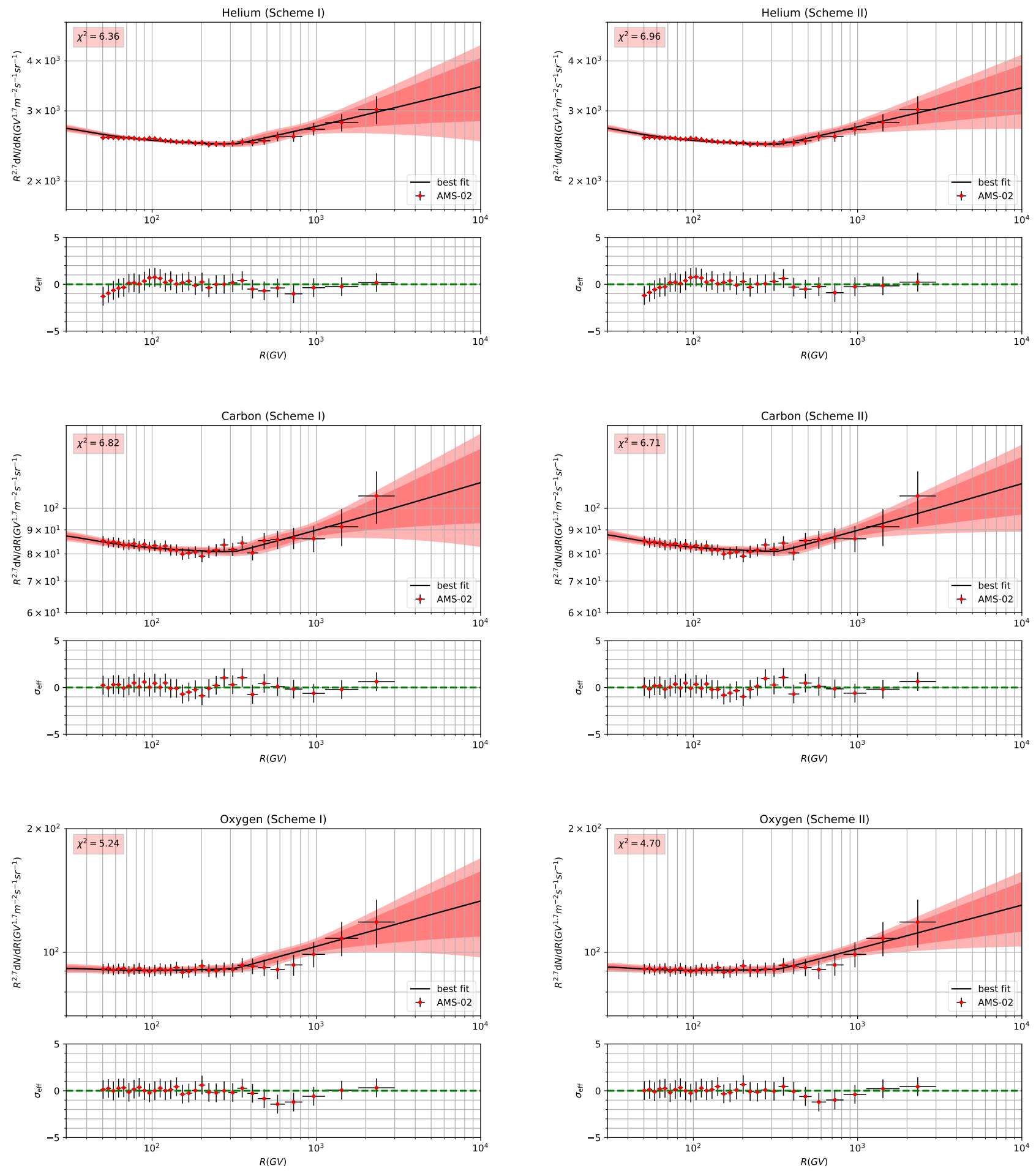

FIG. 2. The global fitting results of the primary $\mathrm{CR}$ nuclei spectra (He, C, and O) for two schemes. The $2 \sigma$ (deep red) and $3 \sigma$ (light red) bounds are also shown in the sub-figures. The relevant $\chi^{2}$ of each nuclei species is given in the sub-figures as well. 

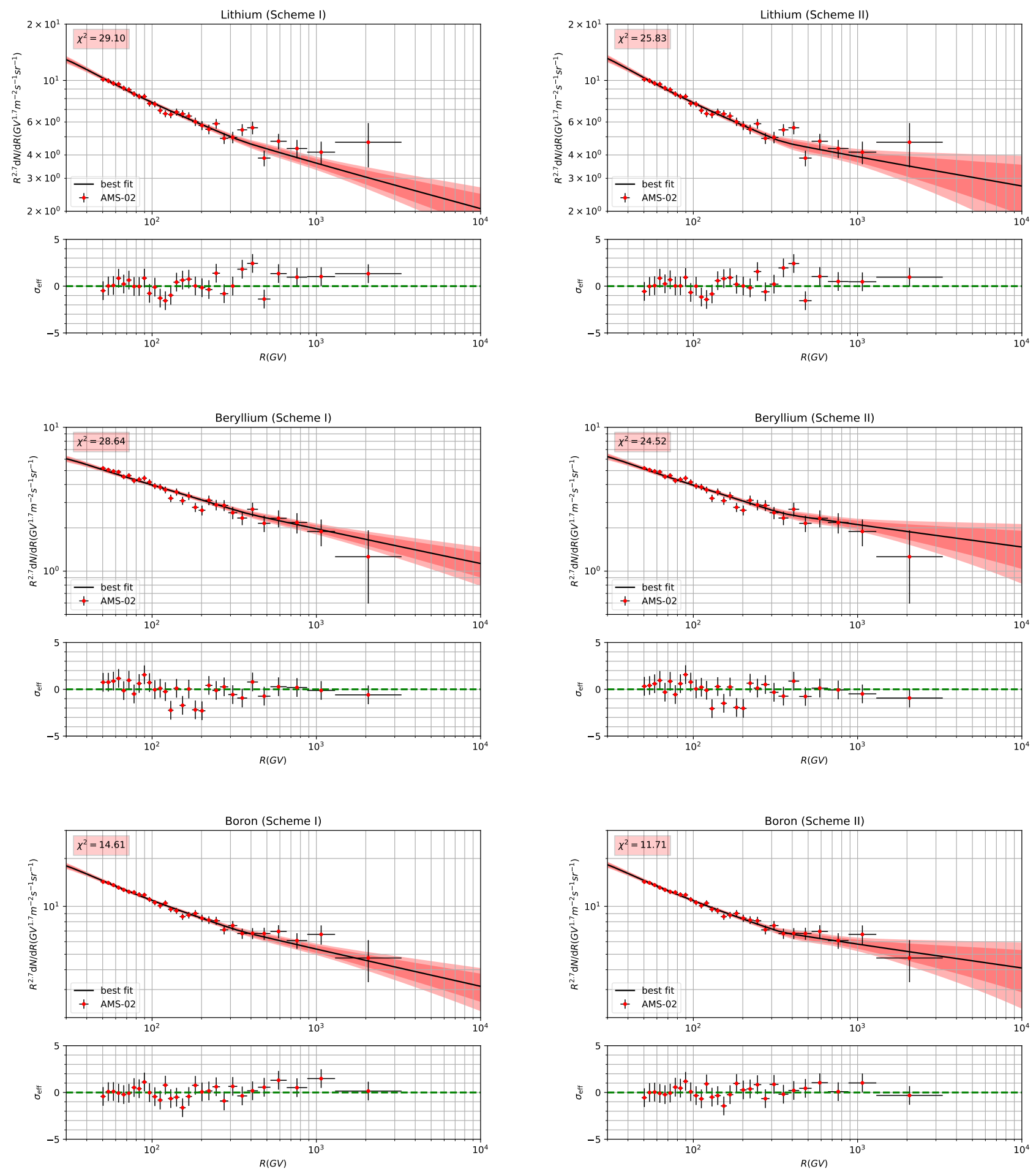

FIG. 3. Same as Fig. 2, but for secondary nuclei species (Li, Be, and B).

extra injection of secondary CR particles in high energy region. All the related details need more attention in future research.

\section{ACKNOWLEDGMENTS}

Jia-Shu Niu would like to appreciate Yi-Hang Nie and Jiu-Qin Liang for their trust and support. This re- 

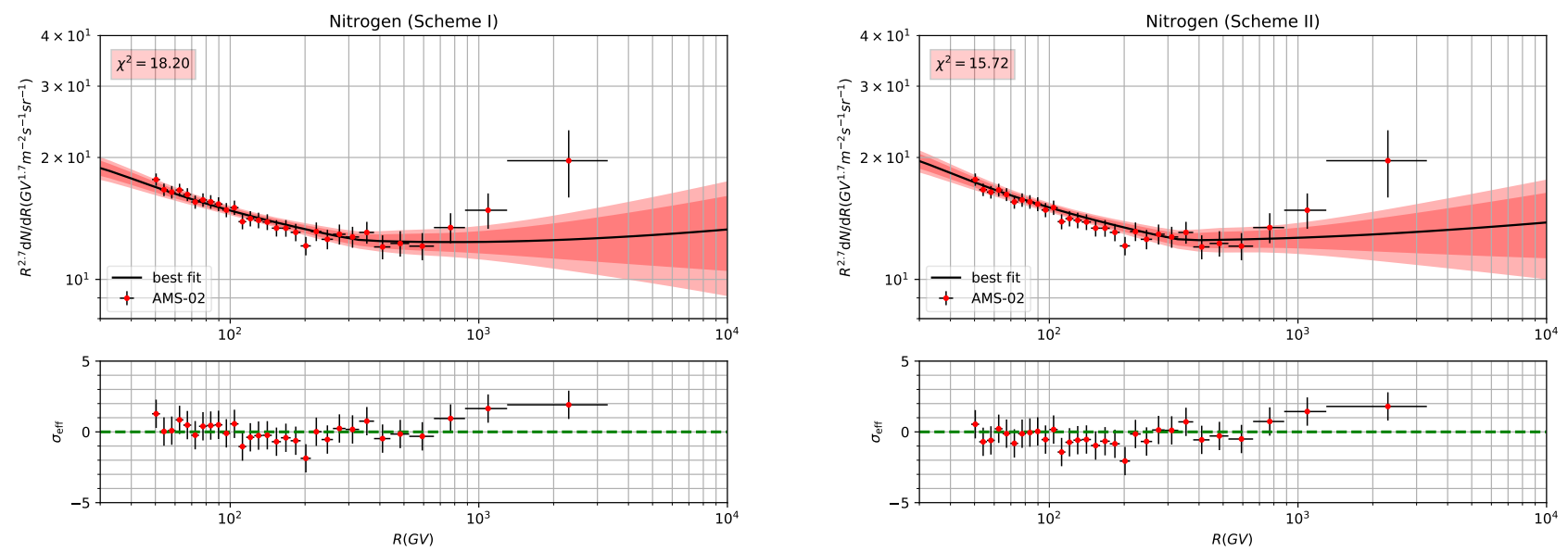

FIG. 4. Same as Fig. 2 but for hybrid nuclei species (N).
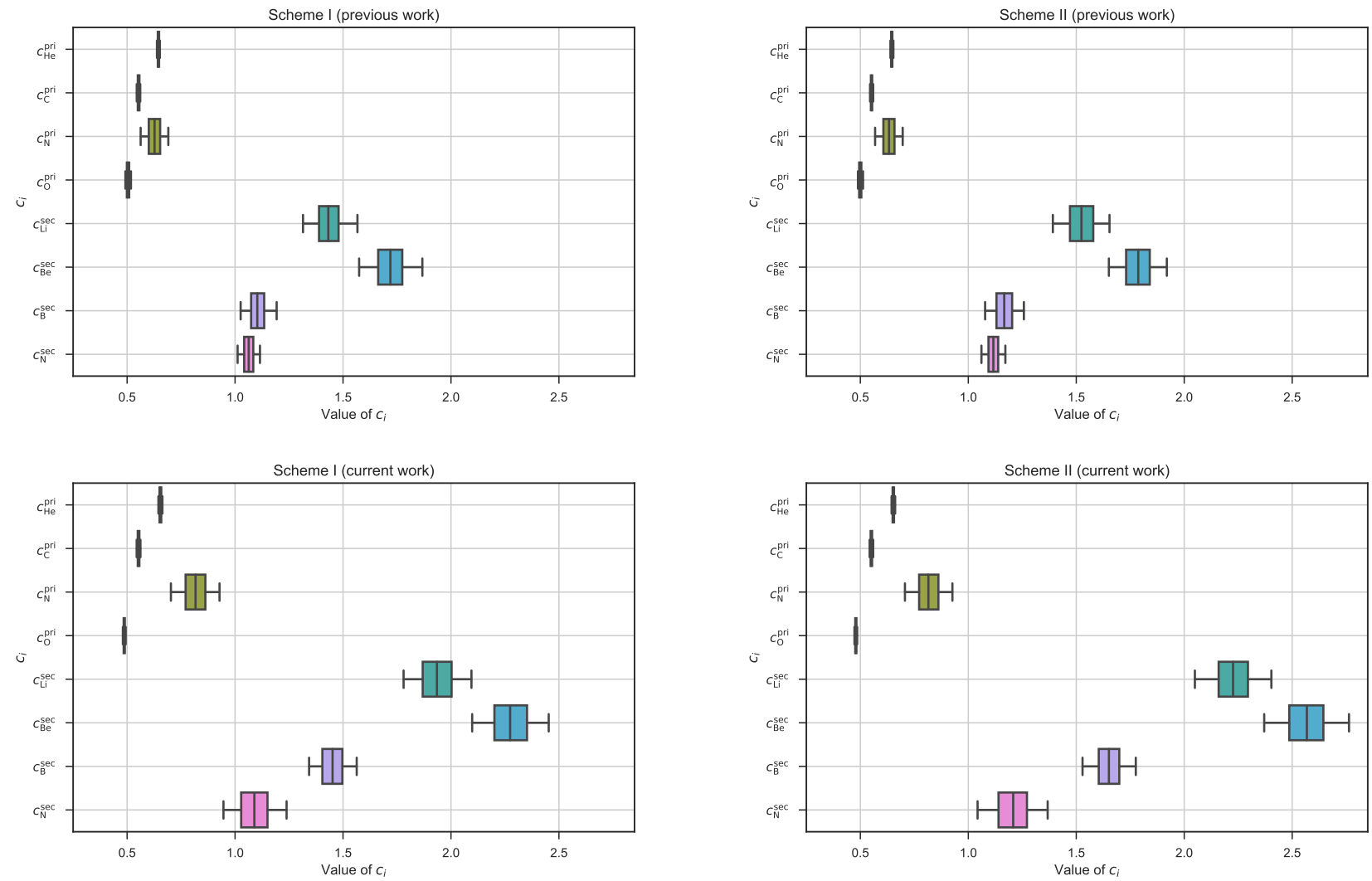

FIG. 5. The boxplot for the re-scale factors of the primary and secondary components in CR nuclei species in Scheme I and II. The upper panels represent the results of our previous work which employed the AMS-02 nuclei data covering from $\sim 2 \mathrm{GV}$ to $\sim 2 \mathrm{TV}$, and the lower panels represent the results of this work which employ the AMS-02 nuclei data larger than $50 \mathrm{GV}$.

search was supported by the Special Funds for Theoretical Physics in National Natural Science Foundation of China (NSFC) (No. 11947125) and the Applied Basic Research Programs of Natural Science Foundation of Shanxi Province (No. 201901D111043). 
[1] J.-S. Niu and T. Li, "Galactic cosmic-ray model in the light of AMS-02 nuclei data," Phys. Rev. D 97, 023015 (2018), arXiv:1705.11089 [astro-ph.HE]

[2] A. D. Panov, J. H. Adams, H. S. Ahn, G. L. Bashindzhagyan, K. E. Batkov, J. Chang, M. Christl, A. R. Fazely, O. Ganel, R. M. Gunashingha, T. G. Guzik, J. Isbert, K. C. Kim, E. N. Kouznetsov, M. I. Panasyuk, W. K. H. Schmidt, E. S. Seo, N. V. Sokolskaya, John W. Watts, J. P. Wefel, J. Wu, and V. I. Zatsepin, "The results of ATIC-2 experiment for elemental spectra of cosmic rays," arXiv e-prints , astro-ph/0612377 (2006), arXiv:astro-ph/0612377 [astro-ph]

[3] H. S. Ahn, P. Allison, M. G. Bagliesi, J. J. Beatty, G. Bigongiari, J. T. Childers, N. B. Conklin, S. Coutu, M. A. DuVernois, O. Ganel, J. H. Han, J. A. Jeon, K. C. Kim, M. H. Lee, L. Lutz, P. Maestro, A. Malinin, P. S. Marrocchesi, S. Minnick, S. I. Mognet, J. Nam, S. Nam, S. L. Nutter, I. H. Park, N. H. Park, E. S. Seo, R. Sina, J. Wu, J. Yang, Y. S. Yoon, R. Zei, and S. Y. Zinn, "Discrepant Hardening Observed in Cosmic-ray Elemental Spectra," Astrophys. J. Lett. 714, L89-L93 (2010), arXiv:1004.1123 [astro-ph.HE]

[4] O. Adriani, G. C. Barbarino, G. A. Bazilevskaya, R. Bellotti, M. Boezio, E. A. Bogomolov, L. Bonechi, M. Bongi, V. Bonvicini, S. Borisov, and el al., "PAMELA Measurements of Cosmic-Ray Proton and Helium Spectra," Science 332, 69 (2011), arXiv:1103.4055 [astro-ph.HE].

[5] M. Aguilar, D. Aisa, B. Alpat, A. Alvino, G. Ambrosi, K. Andeen, L. Arruda, N. Attig, P. Azzarello, A. Bachlechner, and et al., "Precision Measurement of the Proton Flux in Primary Cosmic Rays from Rigidity 1 GV to $1.8 \mathrm{TV}$ with the Alpha Magnetic Spectrometer on the International Space Station," Physical Review Letters 114, 171103 (2015)

[6] M. Aguilar, D. Aisa, B. Alpat, A. Alvino, G. Ambrosi, K. Andeen, L. Arruda, N. Attig, P. Azzarello, A. Bachlechner, and et al., "Precision Measurement of the Helium Flux in Primary Cosmic Rays of Rigidities 1.9 GV to $3 \mathrm{TV}$ with the Alpha Magnetic Spectrometer on the International Space Station," Physical Review Letters 115, 211101 (2015)

[7] M. Aguilar, L. Ali Cavasonza, B. Alpat, G. Ambrosi, L. Arruda, N. Attig, S. Aupetit, P. Azzarello, A. Bachlechner, F. Barao, and et al. (AMS Collaboration), "Observation of the identical rigidity dependence of he, $\mathrm{c}$, and o cosmic rays at high rigidities by the alpha magnetic spectrometer on the international space station," Phys. Rev. Lett. 119, 251101 (2017)

[8] M. Aguilar, L. Ali Cavasonza, G. Ambrosi, L. Arruda, N. Attig, S. Aupetit, P. Azzarello, A. Bachlechner, F. Barao, and et al. (AMS Collaboration), "Observation of new properties of secondary cosmic rays lithium, beryllium, and boron by the alpha magnetic spectrometer on the international space station," Phys. Rev. Lett. 120, 021101 (2018)

[9] In CR physics, nitrogen spectrum is thought to contain both primary and secondary components.

[10] M. Aguilar, L. Ali Cavasonza, B. Alpat, G. Ambrosi, L. Arruda, N. Attig, S. Aupetit, P. Azzarello, A. Bachlechner, F. Barao, and et al. (AMS Collaboration), "Precision measurement of cosmic-ray nitrogen and its pri- mary and secondary components with the alpha magnetic spectrometer on the international space station," Phys. Rev. Lett. 121, 051103 (2018)

[11] Michael Korsmeier and Alessandro Cuoco, "Galactic cosmic-ray propagation in the light of AMS-02: Analysis of protons, helium, and antiprotons," Phys. Rev. D 94, 123019 (2016), arXiv:1607.06093 [astro-ph.HE].

[12] M. J. Boschini, S. Della Torre, M. Gervasi, D. Grandi, G. Jóhannesson, M. Kachelriess, G. La Vacca, N. Masi, I. V. Moskalenko, E. Orlando, S. S. Ostapchenko, S. Pensotti, T. A. Porter, L. Quadrani, P. G. Rancoita, D. Rozza, and M. Tacconi, "Solution of Heliospheric Propagation: Unveiling the Local Interstellar Spectra of Cosmic-ray Species," Astrophys. J. 840, 115 (2017), arXiv:1704.06337 [astro-ph.HE]

[13] J.-S. Niu, T. Li, R. Ding, B. Zhu, H.-F. Xue, and Y. Wang, "Bayesian analysis of the break in DAMPE lepton spectra," Phys. Rev. D 97, 083012 (2018), arXiv:1712.00372 [astro-ph.HE]

[14] Jia-Shu Niu, Tianjun Li, and Fang-Zhou Xu, "A simple and natural interpretations of the dampe cosmic-ray electron/positron spectrum within two sigma deviations," The European Physical Journal C 79, 125 (2019).

[15] C.-R. Zhu, Q. Yuan, and D.-M. Wei, "Studies on CosmicRay Nuclei with Voyager, ACE, and AMS-02. I. Local Interstellar Spectra and Solar Modulation," Astrophys. J. 863, 119 (2018), arXiv:1807.09470 [astro-ph.HE]

[16] J.-S. Niu, T. Li, and H.-F. Xue, "Bayesian Analysis of the Hardening in AMS-02 Nuclei Spectra," Astrophys. J. 873, 77 (2019), arXiv:1810.09301 [astro-ph.HE].

[17] Y. Génolini, P. D. Serpico, M. Boudaud, S. Caroff, V. Poulin, L. Derome, J. Lavalle, D. Maurin, V. Poireau, S. Rosier, P. Salati, and M. Vecchi, "Indications for a High-Rigidity Break in the Cosmic-Ray Diffusion Coefficient," Physical Review Letters 119, 241101 (2017)

[18] P. Blasi, E. Amato, and P. D. Serpico, "Spectral Breaks as a Signature of Cosmic Ray Induced Turbulence in the Galaxy," Physical Review Letters 109, 061101 (2012), arXiv:1207.3706 [astro-ph.HE].

[19] N. Tomassetti, "Origin of the Cosmic-Ray Spectral Hardening," Astrophys. J. Lett. 752, L13 (2012), arXiv:1204.4492 [astro-ph.HE],

[20] N. Tomassetti, "Origin of the Proton-to-helium Ratio Anomaly in Cosmic Rays," Astrophys. J. Lett. 815, L1 (2015), arXiv:1511.04460 [astro-ph.HE]

[21] N. Tomassetti, "Cosmic-ray protons, nuclei, electrons, and antiparticles under a two-halo scenario of diffusive propagation," Phys. Rev. D 92, 081301 (2015), arXiv:1509.05775 [astro-ph.HE]

[22] J. Feng, N. Tomassetti, and A. Oliva, "Bayesian analysis of spatial-dependent cosmic-ray propagation: Astrophysical background of antiprotons and positrons," Phys. Rev. D 94, 123007 (2016), arXiv:1610.06182 [astroph.HE]

[23] Y.-Q. Guo and Q. Yuan, "Understanding the spectral hardenings and radial distribution of Galactic cosmic rays and Fermi diffuse $\gamma$ rays with spatiallydependent propagation," Phys. Rev. D 97, 063008 (2018), arXiv:1801.05904 [astro-ph.HE]

[24] A. E. Vladimirov, G. Jóhannesson, I. V. Moskalenko, and T. A. Porter, "Testing the Origin of High- 
energy Cosmic Rays," Astrophys. J. 752, 68 (2012) arXiv:1108.1023 [astro-ph.HE]

[25] G. Bernard, T. Delahaye, Y.-Y. Keum, W. Liu, P. Salati, and R. Taillet, "TeV cosmic-ray proton and helium spectra in the myriad model," Astron. Astrophys. 555, A48 (2013), arXiv:1207.4670 [astro-ph.HE]

[26] S. Thoudam and J. R. Hörandel, "Revisiting the hardening of the cosmic ray energy spectrum at TeV energies," Mon. Not. Roy. Astron. Soc. 435, 2532-2542 (2013), arXiv:1304.1400 [astro-ph.HE]

[27] N. Tomassetti and F. Donato, "The Connection between the Positron Fraction Anomaly and the Spectral Features in Galactic Cosmic-ray Hadrons," Astrophys. J. Lett. 803, L15 (2015) arXiv:1502.06150 [astro-ph.HE]

[28] M. Kachelrieß, A. Neronov, and D. V. Semikoz, "Signatures of a Two Million Year Old Supernova in the Spectra of Cosmic Ray Protons, Antiprotons, and Positrons," Physical Review Letters 115, 181103 (2015), arXiv:1504.06472 [astro-ph.HE]

[29] N. Kawanaka and S. Yanagita, "Cosmic-Ray Lithium Production at the Nova Eruptions Followed by a Type Ia Supernova," Physical Review Letters 120, 041103 (2018), arXiv:1707.00212 [astro-ph.HE]

[30] Http://galprop.stanford.edu.

[31] Based on the PYTHON module emcee (http://dan.iel.fm/emcee/).

[32] Ming-Yang Cui, Qiang Yuan, Yue-Lin Sming Tsai, and Yi-Zhong Fan, "Possible dark matter annihilation signal in the ams-02 antiproton data," Phys. Rev. Lett. 118, 191101 (2017)

[33] Alessandro Cuoco, Michael Krämer, and Michael Korsmeier, "Novel dark matter constraints from antiprotons in light of ams-02," Phys. Rev. Lett. 118, 191102 (2017)

[34] M. Aguilar, L. Ali Cavasonza, B. Alpat, G. Ambrosi, L. Arruda, N. Attig, S. Aupetit, P. Azzarello, A. Bachlechner, F. Barao, and et al. (AMS Collaboration), "Observation of fine time structures in the cosmic proton and helium fluxes with the alpha magnetic spectrometer on the international space station," Phys. Rev. Lett. 121, 051101 (2018)

[35] M. E. Wiedenbeck, N. E. Yanasak, A. C. Cummings, A. J. Davis, J. S. George, R. A. Leske, R. A. Mewaldt, E. C. Stone, P. L. Hink, M. H. Israel, M. Lijowski, E. R. Christian, and T. T. von Rosenvinge, "The Origin of Primary Cosmic Rays: Constraints from ACE Elemental and Isotopic Composition Observations," Space Sci. Rev. 99, 15-26 (2001).

[36] M. E. Wiedenbeck, W. R. Binns, A. C. Cummings, G. A. de Nolfo, M. H. Israel, R. A. Leske, R. A. Mewaldt, R. C. Ogliore, E. C. Stone, and T. T. von Rosenvinge, "Primary and secondary contributions to arriving abundances of cosmic-ray nuclides," International Cosmic Ray Conference 2, 149-152 (2008).

[37] G. Jóhannesson, R. Ruiz de Austri, A. C. Vincent, I. V. Moskalenko, E. Orlando, T. A. Porter, A. W. Strong, R. Trotta, F. Feroz, P. Graff, and M. P. Hobson, "Bayesian Analysis of Cosmic Ray Propagation: Evidence against Homogeneous Diffusion," Astrophys. J. 824, 16 (2016) arXiv:1602.02243 [astro-ph.HE].

[38] In GALPROP, the abundance of proton is fixted to be a value of $10^{6}$, and all the values in the parenthesis represent the relative abundances to that of proton.

[39] Su-Jie Lin, Qiang Yuan, and Xiao-Jun Bi, "Quantitative study of the ams-02 electron/positron spectra: im- plications for the pulsar and dark matter properties," Physical Review D 91, 063508 (2015), arXiv:1409.6248 [astro-ph.HE]

[40] S.-J. Lin, X.-J. Bi, J. Feng, P.-F. Yin, and Z.-H. Yu, "Systematic study on the cosmic ray antiproton flux," Phys. Rev. D 96, 123010 (2017), arXiv:1612.04001 [astro-ph.HE]

[41] L. C. Tan and L. K. Ng, "Parametrisation of hadron inclusive cross sections in p-p collisions extended to very low energies," Journal of Physics G Nuclear Physics 9, 1289-1308 (1983)

[42] R. P. Duperray, C.-Y. Huang, K. V. Protasov, and M. Buénerd, "Parametrization of the antiproton inclusive production cross section on nuclei," Phys. Rev. D 68, 094017 (2003), astro-ph/0305274.

[43] R. Kappl and M. W. Winkler, "The cosmic ray antiproton background for AMS-02," J. Cosmol. Astropart. Phys. 9, 051 (2014), arXiv:1408.0299 [hep-ph]

[44] M. Di Mauro, F. Donato, N. Fornengo, R. Lineros, and A. Vittino, "Interpretation of AMS-02 electrons and positrons data," J. Cosmol. Astropart. Phys. 4, 006 (2014), arXiv:1402.0321 [astro-ph.HE]

[45] In Bayesian terms, the criterion of a decisive evidence between 2 models is $\Delta \chi^{2} \geq 10$ (see, e.g., Génolini et al. [17]).

[46] A box plot or boxplot is a method for graphically depicting groups of numerical data through their quartiles. In our configurations, the band inside the box shows the median value of the dataset, the box shows the quartiles, and the whiskers extend to show the rest of the distribution which are edged by the 5 th percentile and the 95 th percentile.

[47] Qiang Yuan, Su-Jie Lin, Kun Fang, and Xiao-Jun Bi, "Propagation of cosmic rays in the AMS-02 era," Phys. Rev. D 95, 083007 (2017), arXiv:1701.06149 [astroph.HE]

[48] A. U. Abeysekara, A. Albert, R. Alfaro, C. Alvarez, J. D. Álvarez, R. Arceo, J. C. Arteaga-Velázquez, D. Avila Rojas, H. A. Ayala Solares, A. S. Barber, N. Bautista-Elivar, A. Becerril, E. Belmont-Moreno, S. Y. BenZvi, D. Berley, A. Bernal, J. Braun, C. Brisbois, K. S. Caballero-Mora, T. Capistrán, A. Carramiñana, S. Casanova, M. Castillo, U. Cotti, J. Cotzomi, S. Coutiño de León, C. De León, E. De la Fuente, B. L. Dingus, M. A. DuVernois, J. C. Díaz-Vélez, R. W. Ellsworth, K. Engel, O. EnríquezRivera, D. W. Fiorino, N. Fraija, J. A. García-González, F. Garfias, M. Gerhardt, A. González Muñoz, M. M. González, J. A. Goodman, Z. Hampel-Arias, J. P. Harding, S. Hernández, A. Hernández-Almada, J. Hinton, B. Hona, C. M. Hui, P. Hüntemeyer, A. Iriarte, A. JardinBlicq, V. Joshi, S. Kaufmann, D. Kieda, A. Lara, R. J. Lauer, W. H. Lee, D. Lennarz, H. León Vargas, J. T. Linnemann, A. L. Longinotti, G. Luis Raya, R. LunaGarcía, R. López-Coto, K. Malone, S. S. Marinelli, O. Martinez, I. Martinez-Castellanos, J. MartínezCastro, H. Martínez-Huerta, J. A. Matthews, P. Mirand a-Romagnoli, E. Moreno, M. Mostafá, L. Nellen, M. Newbold, M. U. Nisa, R. Noriega-Papaqui, R. Pelayo, J. Pretz, E. G. Pérez-Pérez, Z. Ren, C. D. Rho, C. Rivière, D. Rosa-González, M. Rosenberg, E. RuizVelasco, H. Salazar, F. Salesa Greus, A. Sand oval, M. Schneider, H. Schoorlemmer, G. Sinnis, A. J. Smith, R. W. Springer, P. Surajbali, I. Taboada, O. Tibolla, 
K. Tollefson, I. Torres, T. N. Ukwatta, G. Vianello, T. Weisgarber, S. Westerhoff, I. G. Wisher, J. Wood, T. Yapici, G. Yodh, P. W. Younk, A. Zepeda, H. Zhou, F. Guo, J. Hahn, H. Li, and H. Zhang, "Extended gamma-ray sources around pulsars constrain the origin of the positron flux at Earth," Science 358, 911-914 (2017), arXiv:1711.06223 [astro-ph.HE].

[49] Gudlaugur Jóhannesson, Troy A. Porter, and Igor V. Moskalenko, "Cosmic-Ray Propagation in Light of the Recent Observation of Geminga," Astrophys. J. 879, 91 (2019), arXiv:1903.05509 [astro-ph.HE]

[50] Kun Fang, Xiao-Jun Bi, Peng-Fei Yin, and Qiang Yuan, "Two-zone Diffusion of Electrons and Positrons from Geminga Explains the Positron Anomaly," Astrophys. J. 863, 30 (2018), arXiv:1803.02640 [astro-ph.HE]

[51] Kun Fang, Xiao-Jun Bi, and Peng-Fei Yin, "Reanalysis of the Pulsar Scenario to Explain the Cosmic Positron Excess Considering the Recent Developments," Astrophys. J. 884, 124 (2019), arXiv:1906.08542 [astroph.HE],
[52] Kun Fang, Xiao-Jun Bi, and Peng-Fei Yin, "Possible origin of the slow-diffusion region around Geminga," Mon. Not. Roy. Astron. Soc. 488, 4074-4080 (2019), arXiv:1903.06421 [astro-ph.HE]

[53] Of course, it might come from the imperfect model on solar modulation in our previous work in low energy region $(R<50 \mathrm{GV})$.

[54] Ruizhi Yang and Felix Aharonian, "Interpretation of the excess of antiparticles within a modified paradigm of galactic cosmic rays," Phys. Rev. D 100, 063020 (2019) arXiv:1812.04364 [astro-ph.HE].

[55] M. J. Boschini, S. Della Torre, M. Gervasi, D. Grand i, G. Johannesson, G. La Vacca, N. Masi, I. V. Moskalenko, S. Pensotti, T. A. Porter, L. Quadrani, P. G. Rancoita, D. Rozza, and M. Tacconi, "Deciphering the local Interstellar spectra of secondary nuclei with GALPROP/HelMod framework and a hint for primary lithium in cosmic rays," arXiv e-prints , arXiv:1911.03108 (2019), arXiv:1911.03108 [astro-ph.HE] 